\section{Kompass Ophthalmologie}

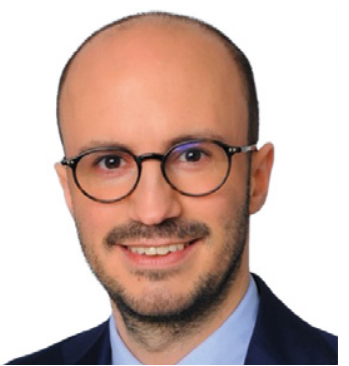

\section{Dr. Alexander C. Rokohl}

Uniklinik Köln - Centrum für Integrierte Onkologie (CIO) Köln, Köln-Lindenthal, Deutschland

\title{
7 Fragen an
}

\section{Dr. Alexander C. Rokohl,}

\section{Träger des DOG Wissenschaftspreises Trockenes Auge und Blepharitis/MGD 2021}

\section{Akademischer Werdegang}

- 2011-2017: Studium der Humanmedizin an der Universität zu Köln

- 2018: Promotion zum Dr. med. (magna cum laude) an der Universität zu Köln

- Seit Juni 2018: Assistenzarzt am Zentrum für Augenheilkunde, Universitätsklinikum Köln

- Seit Juni 2019: Clinician-Scientist an der Universität zu Köln

\section{Forschungsschwerpunkte}

- Dry Anophthalmic Socket Syndrome (DASS)

- Augenprothetik

- Psychologische Aspekte sowie körperliche und funktionelle Adaption und Kompensation nach Enukleation

- Optimierung der Augenlidchirurgie, der minimal-invasiven Tränenwegchirurgie und der Orbitachirurgie

- Dreidimensionale Anthropometrie der periokulären Region

- Untersuchung von molekularen und zellulären Metastasierungsmechanismen bei okulären Tumoren

Auf dem DOG-Kongress 2021 wurden Sie für Ihre Forschung zum sogenannten «Dry Anophthalmic Socket Syndrome» mit dem Wissenschaftspreis Trockenes Auge und Blepharitis/MGD ausgezeichnet. Welche Ziele lagen Ihren Arbeiten zugrunde? Was untersuchten Sie?

Es erscheint durchaus erstaunlich, dass ein Wissenschaftspreis «Trockenes Auge» an ein Projekt verliehen wurde, bei dem das Auge bereits entfernt ist (Anophthalmus). Aber gerade diese anophthalmischen Patienten beklagen massive Trockenheitsbeschwerden, teilweise sogar schwerer als Patienten mit Trockenem Auge. Deshalb hat sich unsere Arbeitsgruppe unter Leitung von Herrn Univ.-Prof. Dr. Dr. L. M. Heindl in Köln der Erforschung des anophthalmischen Sockets verschrieben. Das Ziel unserer Arbeiten war es, die Beschwer- 
den anophthalmischer Patienten standardisiert zu erheben, die klinischen Befunde zu quantifizieren und alles in einen sinnvollen Kontext zu bringen, um das Dry Anophthalmic Socket Syndrome erstmals zu definieren. Insbesondere haben wir die klinische Entzündungsreaktion sowie die Morphologie der Meibomdrüsen am anophthalmischen Socket untersucht.

\section{Zu welchen Ergebnissen sind Sie gekommen?}

Auf Basis der Projektergebnisse und in Zusammenschau mit den Ergebnissen aus Vorstudien konnten wir in unserer hochrangigen Publikation «Dry Anophthalmic Socket Syndrome - Standardized clinical evaluation of symptoms and signs» ein neues Krankheitsbild, das «Dry Anophthalmic Socket Syndrome (DASS)», weltweit zum ersten Mal beschreiben, definieren und auch Diagnostikkriterien erstellen. Die Publikation «Dry anophthalmic socket syndrome - morphological alterations in meibomian glands» zeigt auf, dass es nach der Entfernung eines Auges und durch das Prothesentragen zu morphologischen Veränderungen der Meibomdrüsen am anophthalmischen Socket kommt.

Was hat Sie während der Arbeit oder nach Auswertung der Ergebnisse überrascht?

Bei der Arbeit mit Augenprothesenträgern überrascht uns immer wieder, dass es einen sehr großen Leidensdruck in vielerlei Hinsicht - z.B. auch durch psychosoziale Belastungen - gibt, jedoch gibt es kaum evidenzbasierte Therapien und Versorgungsstrukturen für diese Probleme. Hier besteht einerseits höchster Forschungsbedarf und andererseits muss durch Translation der Forschungsergebnisse die Versorgungsqualität deutlich verbessert werden.

Was könnte Ihre Forschung für die praktische, klinische Arbeit eines Ophthalmologen bedeuten?

Das DASS, welches bei der Mehrheit der Patienten nach einer Enukleation oder einer Eviszeration auftritt, reduziert, ähnlich wie das trockene Auge, die Lebensqualität dieser Patienten signifikant.
Die Erkenntnisse aus unseren Publikationen zum DASS sind bereits heute schon essentiell in der klinischen Beurteilung und Versorgung anophthalmischer Patienten und tragen maßgeblich zu einer Optimierung der augenprothetischen Rehabilitation mit Verbesserung der Lebensqualität bei.

\section{Wofür setzen Sie das Förderungsgeld ein?}

Die erhaltenen Fördergelder werden zur weiteren Intensivierung der Forschung im Bereich der Augenprothetik eingesetzt.

\section{An was forschen Sie im Moment? Was ist Ihr nächstes Ziel?}

Unsere bisherigen Publikationen zum DASS haben den Grundstein für diverse weitere Studien gelegt. Insbesondere die Entwicklung einer evidenzbasierten Behandlung nach einem Stufenschema für das DASS konnte durch diese Publikationen initiiert werden.

Womit schaffen Sie für sich selbst und für Ihr Umfeld einen Ausgleich zu Ihrem Einsatz für die Forschung?

Zum Ausgleich spiele ich Tennis sowie Fußball, treffe mich regelmäßig mit meinen Freunden und engagiere mich ehrenamtlich in verschiedenen Vereinen.

Haben Sie ganz herzlichen Dank für das Interview, Herr Dr. Rokohl!

\section{ANGABEN ZUM PREIS}

Der DOG-Wissenschaftspreis Trockenes Auge und Blepharitis/ MGD gestiftet durch die Firma Optima Pharmazeutische GmbH wird jährlich auf dem Jahreskongress der Deutschen Ophthalmologischen Gesellschaft verliehen. Ziel des Preises ist es, herausragende klinische und grundwissenschaftliche Arbeiten zum Trockenen Auge und Blepharitis/Meibom-Drüsen Dysfunktion (MGD) auszuzeichnen. Der Preis ist mit $3000 €$ dotiert. 2021 wurde der Preis zu gleichen Teilen an Herrn Dr. Alexander C. Rokohl (Köln) und Herrn PD Dr. Martin Schicht (Erlangen) verliehen. 